\title{
Acetylation site specificities of lysine deacetylase inhibitors in human
}

\section{cells}

Christian Schölz ${ }^{1}$, Brian T. Weinert ${ }^{1}$, Sebastian A. Wagner ${ }^{1}$, Petra Beli ${ }^{1}$, Yasuyuki Miyake ${ }^{2}$, Jun $\mathrm{Qi}^{3}$, Lars J. Jensen ${ }^{4}$, Werner Streicher ${ }^{5}$, Anna R. McCarthy ${ }^{6,}$, Nicholas J. Westwood ${ }^{7}$, Sonia Lain ${ }^{6}$, Jürgen Cox $^{8}$, Patrick Matthias ${ }^{2}$, Matthias Mann ${ }^{8}$, James E. Bradner ${ }^{3}$, Chunaram Choudhary ${ }^{1, *}$ ${ }^{1}$ Department of Proteomics, The Novo Nordisk Foundation Center for Protein Research, Faculty of Health Sciences, University of Copenhagen, Copenhagen, Denmark

${ }^{2}$ Friedrich Miescher Institute for Biomedical Research, Basel, Switzerland

${ }^{3}$ Department of Medical Oncology, Dana-Farber Cancer Institute, Harvard Medical School, Boston, USA ${ }^{4}$ Department of Protein Science and Technology, The Novo Nordisk Foundation Center for Protein Research, Faculty of Health Sciences, University of Copenhagen, Copenhagen, Denmark

${ }^{5}$ Department of Disease Systems Biology, The Novo Nordisk Foundation Center for Protein Research, Faculty of Health Sciences, University of Copenhagen, Copenhagen, Denmark

${ }^{6}$ Department of Microbiology, Tumor and Cell biology, Karolinska Institutet, Stockholm, Sweden

${ }^{7}$ School of Chemistry and Biomedical Sciences Research Complex, EaStCHEM, University of St Andrews, St Andrews, Scotland, UK

${ }^{8}$ Department of Proteomics and Signal Transduction, Max Planck Institute for Biochemistry, Martinsried,

Germany

†author is deceased

*Correspondence should be addressed to:

Chunaram Choudhary

Email: chuna.choudhary@cpr.ku.dk

Running title: In-vivo target profiling of lysine deacetylase inhibitors

Key words: Acetylation, deacetylase inhibitor, SILAC, HDAC, KDAC, mass spectrometry, proteomics, sirtuin, nicotinamide, tenovin-6, bufexamac

\section{Abstract}


Lysine deacetylases inhibitors (KDACIs) are used in basic research, and many are being investigated in clinical trials for treatment of cancer and other diseases. However, their specificities in cells are incompletely characterized. Here we used quantitative mass spectrometry to obtain acetylation signatures for 19 different KDACIs, covering all 18 human lysine deacetylases. Most KDACIs increased acetylation of a small, specific subset of the acetylome, including sites on histones and other chromatin-associated proteins. Inhibitor treatment combined with genetic deletion showed that the effects of the pan-sirtuin inhibitor nicotinamide are primarily mediated by SIRT1 inhibition.

Furthermore, we confirmed that the effects of tubacin and bufexamac on cytoplasmic proteins result from inhibition of HDAC6. Bufexamac also triggered an HDAC6-independent hypoxia-like response by stabilizing HIF1- $\alpha$, providing a possible mechanistic explanation of its adverse, pro-inflammatory effects. Our results offer a systems view of KDACI specificities, providing a framework for studying function of acetylation and deacetylases. 


\section{Intro}

Lysine acetylation is a reversible posttranslational modification that is controlled by the opposing activities of lysine acetyltransferases and deacetylases. The human genome encodes 18 different lysine deactetylases (KDACs), which play important regulatory roles in several biological processes, including gene transcription ${ }^{1}$, silencing ${ }^{2}$, cytoskeletal reorganization and cell migration ${ }^{3}$ and DNA damage repair ${ }^{4}$. Depending on their sequence homology with yeast deacetylases as well as their domain organization and subcellular localization, KDACs are classified into four different classes (Fig. 1a). The importance of KDACs in mammalian physiology is highlighted by essential roles of many deacetylases in embryonic viability or postnatal development in mouse models ${ }^{5-7}$.

Dysregulation of acetylation has been implicated in various human diseases, and thus KDACs are attractive therapeutic targets ${ }^{8,9}$ for diseases such as viral infections ${ }^{10}$, inflammation ${ }^{11}$, neurodegenerative diseases ${ }^{12}$, metabolic disorders ${ }^{13}$, and cancer ${ }^{9}$. In the past decade, $>400$ clinical trials have been initiated with different KDACIs, leading to the approval of vorinostat (suberoylanilide hydroxamic acid, SAHA) and romidepsin (FK228) for the treatment of cutaneous Tcell lymphoma ${ }^{14}$. The therapeutic potential of KDACIs has encouraged the development of KDACIs with selectivity for different deacetylases, and currently more than a dozen different inhibitors are being tested in clinical trials ${ }^{14-17}$.

The specificities of KDACIs have been extensively determined at the level of the enzyme that they target, often using in-vitro deacetylation assays with recombinant deacetylases ${ }^{18}$ or chemical proteomics-based approaches ${ }^{19,20}$. However, these methods do not reveal KDACI specificity in cells at the level of individual acetylation sites, which is critical for understanding their distinct modes of action and to stimulate scientific investigation and elucidate new therapeutic opportunities.

Here we applied quantitative mass spectrometry (MS) to evaluate the specificities of 19 structurallydivergent KDACIs at the level of acetylation sites affected in human cells. We quantified acetylation at more than 8,000 sites in inhibitor-treated HeLa cells and used genetic KDAC knockout cells to confirm the predicted specificities of several prominent KDACIs.

\section{Results}

\section{Strategy for KDACI-regulated acetylome analysis}


We selected 19 KDACIs that are widely used as research tools or drugs, and cover the entire selectivity range for human deacetylases, albeit with varying specificity profiles, as determined in previous studies using in-vitro deacetylation assays $s^{18-20}$ (Fig. 1a). In addition, we included a highly selective KDAC1 and 2 inhibitor, JQ12, which is an emerging chemical tool for studying isoformselective inhibition of class I KDACs (Supplemental note 1 and Supplemental Fig. 1a-c).

To evaluate the selectivity of KDACIs for acetylation sites in cells, we used a stable isotope labeling by amino acids in cell culture (SILAC)-based quantitative mass spectrometry (MS) approach ${ }^{21}$. Human cervical cancer (HeLa) cells were metabolically labeled with different stable isotopic forms of lysine and arginine ( $\mathrm{Lys}^{0}$ and $\mathrm{Arg}^{0}$, "light" SILAC; or Lys ${ }^{8}$ and $\mathrm{Arg}^{10}$, "heavy" SILAC). The "heavy" labeled cells were treated for 16 hours with the respective KDACIs, and their effects on acetylation levels were determined by comparison with "light" labeled control cells that were mocktreated with solvent (DMSO or $\mathrm{H}_{2} \mathrm{O}$ ) (inhibitor concentrations are provided in Supplemental Table 1). Acetylated peptides were enriched using anti-acetyllysine antibodies and analyzed by high resolution mass spectrometry ${ }^{22}$ (Fig. 1b).

\section{An overview of the KDACI acetylation screen}

For each of the KDACIs, site-specific acetylation changes were quantified in two independent biological replicates, which had an average Pearson correlation coefficient of 0.71 (Supplemental Fig. 2), supporting the overall quantitative reliability of the approach. Altogether, the dataset contains $>8,100$ quantified acetylation sites in HeLa cells (Supplemental Table 2), covering $~ 80 \%$ of previous identified $\operatorname{sites}^{23}$ (Supplemental Fig. 3), which were localized to the indicated lysines with greater than $90 \%$ confidence (average site localization probability of 0.99$)$. A majority ( 85\%) of the acetylation sites was quantified in more than one KDACI-treated sample, and nearly $60 \%$ of the sites were quantified in response to five or more KDACIs (Supplemental Table 2).

We quantified an average of >3,200 acetylation sites for each of the analyzed KDACIs (Fig. 1c), enabling us to assess in cells the inhibitory scope and acetylation pattern for each of the tested compounds. For further analyses, we set a threshold of a greater than two-fold increase or decrease in acetylation for regulated acetylation sites. Notably, most inhibitors increased acetylation of only a small fraction of the quantified sites (on average 6.2\%) above this threshold (Fig. 1c), demonstrating that a majority of KDACIs have a limited, though specific, inhibitory scope at the acetylation site level. The activity of KDACs diminishes protein acetylation levels; accordingly, for all investigated 
inhibitors except sirtinol, the fraction of upregulated acetylation sites in KDACI-treated cells was greater than the fraction of downregulated sites (Fig. 1c).

\section{Specificity of KDACIs for acetylation sites}

To understand the specificity patterns of KDACIs in cells, we used average linkage clustering to group KDACIs based on their effects on the hyperacetylation of sites in cells. Many of the classical histone deacetylase inhibitors, such as LBH589, PXD101, SAHA, TSA, grouped together, and this group was closely related to the group of KDACIs targeting class I deacetylases including apicidin, MS275, CI.994, MGCD0103, and valproic acid (VPA) (Fig. 2a). The acetylation specificity profiles of many of these inhibitors in cells (i.e., apicidin, MS275, CI.994, MGCD0103) are consistent with their described deacetylase inhibitory profiles in cell-free systems. However, the inhibitory profiles of several KDACIs, such as tenovin-6, sirtinol and nicotinomide (NAM), were distinct from each other and from the remaining KDACIs.

We examined the subcellular distribution of proteins with KDACI-upregulated acetylation sites by association of acetylated proteins with the Gene Ontology (GO) terms "Nuclear", "Cytoplasm", and "Mitochondrial" (Fig. 2b). A majority of inhibitors caused increased acetylation of nuclear proteins and for eight of the inhibitors, more than $60 \%$ of upregulated acetylation sites were present on nuclear proteins (compared to $30 \%$ of all sites). Acetylation of $\mathrm{N}$-terminal lysines in histones has numerous regulatory roles 24,25 . Several inhibitors of Class I and IIa KDACs showed similar regulatory profiles for histones and strongly increased acetylation of $\mathrm{N}$-terminal lysines, whereas other KDACIs such as PCI34051, sirtinol and tenovin-6 did not cause extensive acetylation of the Nterminal histone tails (Fig. 2c). A large number of acetylation sites was also quantified on the middle and C-terminal parts of histones; however, most of these sites were not similarly upregulated by the KDACIs, indicating that histone deacetylases function in a site-selective manner and differentially affect distinct sites on the same proteins.

Tenovin-6, NAM, and tubacin were among the most promiscuous inhibitors, each increasing acetylation at more than ten percent of all quantified sites (Fig. 1c). However, the acetylation profiles of these inhibitors were relatively distant from each other, and from that of most other inhibitors (Fig. 2a). Many acetylation sites were commonly quantified in cells treated with broad-range inhibitors such as tenovin-6, NAM, PCI24781, and tubacin; however, few sites were commonly regulated by these inhibitors (Fig. 2d). Thus, each of these inhibitors affects a large, but distinct, 
subset of acetylation sites. Among these four KDACIs, only tubacin and PCI24781-upregulated sites showed a substantial overlap, consistent with both compounds targeting HDAC6 ${ }_{\Delta}^{26,27}$.

Based on the fraction of upregulated sites, the HDAC8-specific inhibitor PCI34051 ${ }_{\triangle}^{28}$ was the most selective KDAC inhibitor in our screen (Fig. 1c). We identified SMC3 acetylation as a key target of this inhibitor (Supplemental Table 2, Supplemental Fig. 4a, b). Acetylation of lysines K105/K106 of SMC3 is important for the establishment of chromosome cohesion ${ }^{29-31}$. These results confirm a recent study that identified HDAC8 as a SMC3 deacetylase ${ }^{32}$ and indicate that HDAC8, at least in cultured human cells, functions as a highly selective deacetylase targeting only a few proteins.

Considering the broad effects of tubacin, bufexamac, tenovin-6, and NAM, and their distinct acetylation profiles, we decided to further analyze the mechanisms and properties of the acetylation sites and proteins that were affected by these inhibitors.

\section{NAM increases acetylation of nuclear sites}

Sirtuin deacetylases require the cofactor $\mathrm{NAD}^{+}$for deacetylase activity and generate nicotinamide (NAM) from $\mathrm{NAD}^{+}$during the deacetylation reaction. NAM, which noncompetitively inhibits sirtuins without affecting their binding of $\mathrm{NAD}^{+33}$, has been widely used as a pan-sirtuin inhibitor. In our dataset, NAM was among the inhibitors that affected the largest number of sites and increased acetylation more than two-fold at $12 \%$ of sites, and more than 1.5 -fold at $20 \%$ of sites (Fig. 1c), suggesting a broad substrate range for sirtuin deacetylases. NAM-sensitive acetylation sites were predominantly present on nuclear proteins that are involved in diverse biological processes in this compartment (Fig. 2b, 3a). Acetylation of most mitochondrial proteins was not specifically increased even after long-term (72h) treatment with NAM (Supplemental Fig. 5a-c, Supplemental Table 3), despite sirtuins being the only known deacetylases in this organelle (Fig. 2b, Supplemental Table 4). These unexpected results were independently confirmed in human acute myelocytic leukemia cells (MV4-11) (Fig. 3b, Supplemental Table 5).

Among the NAM-upregulated acetylation sites, many were present on known substrates of SIRT1 such as p53, BAZ2a, NPM1, MOF, WRN, Ku70 and Ku80 (Supplemental Tables 4, 5). In addition, many proteins with NAM-upregulated acetylation sites were interconnected in functional networks, and were enriched in Gene Ontology (GO) terms such as transcription, splicing, and DNA damage (Supplemental Fig. 6a).

In order to identify the cellular deacetylases that are targets of NAM, we compared NAM-regulated acetylation in mouse embryonic fibroblasts (MEFs) to acetylation changes caused by deletion of 
Sirt1, Sirt2, or Sirt6 (Supplemental Fig. 6b-c, Supplemental Tables 6-9), which are known to deacetylate proteins in non-mitochondrial compartments. Similar to NAM treatment, and in agreement with a previous study ${ }^{34}$, acetylation was increased at several hundred sites in Sirt $1^{-/}$cells (Supplemental Table 6). The SIRT1- and NAM-regulated acetylomes showed a strong correlation $(\mathrm{R}=0.63$ ) (Fig. 3c), and similar GO terms were enriched in the NAM- and SIRT1-regulated acetylomes (Fig. 3d), suggesting that SIRT1 is the major target of NAM in cells. This is also supported by a good correlation of NAM- and the SIRT1 inhibitor EX-527-regulated acetylomes $(\mathrm{R}=0.68)$ (Supplemental Fig. 7, Supplemental Table 10). In contrast, a smaller fraction of the acetylome changed in $\operatorname{Sirt}^{-/}$and $\operatorname{Sirt}^{-/}$cells compared to Sirt $1^{-/}$cells, and these changes correlated weakly with NAM-induced changes $(\mathrm{R}=0.1$ ) (Supplemental Fig. 6d, 8). SIRT2-regulated acetylation also showed a modest correlation with the SIRT2 inhibitors sirtinol and AGK2 $(\mathrm{R}=0.11$ for sirtinol vs Sirt2 $2^{-/} ; \mathrm{R}=0.35$ for AGK2 vs Sirt2 ${ }^{-/}$) (Supplemental Fig. 8, Supplemental Tables 8, 9), indicating that these inhibitors weakly inhibited SIRT2 in MEFs at the concentrations used in our study.

\section{Tenovin-6 increases mitochondrial acetylation}

We and others have previously identified a large number of acetylation sites on mitochondrial proteins in human cells $\mathrm{s}^{22,35,36}$; however, no KDACI has been demonstrated to selectively increase acetylation of mitochondrial proteins. In our screen, we identified tenovin- 6 as a specific regulator of mitochondrial acetylation (Fig. 2b and Supplemental Fig. 9a). In total, 405 acetylation sites showed elevated acetylation upon tenovin-6 treatment (Supplemental Table 2), and a large majority of these sites (more than $90 \%$ ) was present on proteins annotated as mitochondrial (Supplemental Fig. 9b); corresponding to more than $50 \%$ of all identified mitochondrial acetylation sites in tenovin- 6 treated cells. Several mitochondria-related Gene Ontology (GO) terms, such as ATP synthetase complex, mitochondrial matrix, and mitochondrial lumen, showed significant enrichment among proteins with tenovin-6-upregulated acetylation sites (Supplemental Fig. 9b). Also, enzymes that participate in metabolic pathways, such as the TCA cycle and fatty acid elongation, were significantly enriched within the group of differentially acetylated proteins (Supplemental Fig. 9c,d). These data demonstrate that tenovin-6, an anti-cancer compound, specifically increases acetylation of mitochondrial proteins.

\section{Tubacin and bufexamac inhibit HDAC6}


Tubacin and bufexamac were among the few inhibitors that affected a broad substrate range with similar acetylation changes (Fig. 4a) and increased acetylation of the well-known HDAC6 substrate $\operatorname{cortactin}^{37}$ (Supplemental Tables 2, 11). Tubacin and bufexamac have been reported to be selective for HDAC6 $6_{A}^{19,27}$, and the broad effects on the cellular acetylome we observed prompted us to investigate whether these changes are mediated via HDAC6 or through independent mechanisms. We found that deletion of Hdac6 also affected a large number of acetylation sites in MEF cells, and HDAC6-regulated acetylation changes correlated with those caused by tubacin and bufexamac treatment $\left(\mathrm{R}=0.54\right.$ for bufexamac vs Hdac $^{--} ; \mathrm{R}=0.53$ for Tubacin vs Hdac6 $\left.^{-/}\right)($Fig. $4 \mathrm{~b}, \mathrm{c}$; Supplemental Table 12). Furthermore, similar GO terms were enriched among proteins with increased acetylation in response to inhibitor treatment or deletion of Hdac6 (Fig. 4d). These data showed that a large fraction of bufexamac- and tubacin-regulated acetylation is likely to be caused by inhibition of their endogenous target deacetylase HDAC6.

\section{Bufexamac activates the HIF1- $\alpha$ pathway}

Until recently, bufexamac was used as a non-steroidal anti-inflammatory drug (NSAID) for the treatment of inflammatory dermatoses, but due to growing evidence that it can provoke contact dermatitis, its clinical use was revoked in Europe in $2010^{38}$. The mechanisms by which bufexamac causes inflammation remain unknown.

A majority of bufexamac-based ointments thought to be anti-inflammatory contained very high concentrations of this compound ( $\sim 5 \%$, corresponding to $224 \mathrm{mM})$, whereas it is known that bufexamab efficiently increases acetylation of the HDAC6 target tubulin at 10,000-fold lower concentrations (less than $10 \mu \mathrm{M})^{19}$ (see also Fig. $5 \mathrm{~g}$ ). To understand the possible mechanistic basis of its pro-inflammatory effects, we analyzed changes in lysine acetylation and protein expression in HeLa cells treated with two different concentrations of bufexamac $(50 \mu \mathrm{M}[0.001 \%]$ and $1 \mathrm{mM}$ [0.022 \%]) (Supplemental Fig. 10a). The high dose concentration used here is similar to the lowest doses of bufexamac that are sufficient to cause inflammatory reactions in patients ${ }^{39}$. High-dose bufexamac substantially increased abundance of an acetylated peptide from hypoxia-inducible factor 1 alpha (HIF1- $\alpha$ ) (Supplemental Fig. 10b, Supplemental Table 2). HIF1- $\alpha$ is a transcription factor that plays essential roles in the cellular responses to hypoxia, and acetylation can stabilize HIF1- $\alpha$ protein by preventing its proteasomal degradation ${ }^{40}$. Indeed, HIF1- $\alpha$ was strongly induced by bufexamac and the increase in HIF1- $\alpha$ levels was comparable to commonly used hypoxia mimetics such as ciclopirox olamine (CPX) and deferoxamine (DFX) (Fig. 5a). In contrast to bufexamac, two other well-knows NSAIDs, aspirin and ibuprofen, failed to increase HIF1- $\alpha$ protein levels indicating 
that this effect is specific to bufexamac (Fig. 5b). HIF1- $\alpha$ was rapidly stabilized (Fig. 5c), and accumulated in the nucleus of bufexamac treated cells (Fig. 5d).

Bufexamac readily increased the transcriptional activity of HIF1- $\alpha$ in a luciferase reporter assay (Fig. 5e), as well as increased the expression of its endogenous target gene vascular endothelial growth factor A (VegfA) (Fig. 5f). Furthermore, we analyzed changes in protein expression in bufexamactreated cells by quantifying over 5,800 proteins (Supplemental Table 13). Among 23 proteins that were reproducibly upregulated by high-dose bufexamac in all three experiments, over $70 \%$ were previously known to be upregulated by hypoxia (Supplemental Fig. 10c, Supplemental Table 13). In contrast, none of those proteins were consistently upregulated in the low-dose bufexamac-treated cells. These results confirmed the induction of hypoxia-like responses in bufexamac-treated cells.

Notably, stabilization of HIF1- $\alpha$ occurred only at relatively high doses (>200 $\mu \mathrm{M})$ of bufexamac, whereas increased acetylation of tubulin was already detectable at substantially lower doses $(>5 \mu \mathrm{M})$ (Fig. 5g). These results suggested that bufexamac-induced hypoxia responses may occur independently of its KDAC inhibitory functions. Bufexamac is a hydroxamic acid compound, and many compounds of this class function as metal chelators. Because iron chelators are commonly used as hypoxia mimetics, we surmised that bufexamac may induce hypoxia-like responses by chelating cellular iron. Indeed, supplementation of iron during bufexamac treatment abolished bufexamac-induced HIF1- $\alpha$ protein induction in a dose-dependent manner (Fig. 5h) as well as its transcriptional activity (Fig. 5i). Treatment of iron-dependent HL-60 (Human promyelocytic leukemia cells) cells with bufexamac caused cell death, and this effect was completely reversed by iron substitution (Supplemental Fig. 11). A direct interaction between bufexamac and iron was further shown by colorimetric iron chelation assay using UV/VIS spectroscopy (Supplemental Fig. $12 \mathrm{a}, \mathrm{b})$. Taken together, these results show that at lower doses bufexamac specifically inhibits HDAC6, whereas at higher concentrations it also chelates cellular iron, thereby activating the HIF1$\alpha$ pathway by mimicking hypoxia (Supplemental Fig. 13).

\section{Discussion}

A large number of small molecule KDAC inhibitors has been identified that show distinct selectivity for different deacetylases ${ }^{8,9,16}$. In addition to their clinical use, KDACIs are commonly used in basic research to study function of acetylation and deacetylation. Specificity profiles generated in cell-free systems are frequently used as a reference for selecting appropriate KDACIs. Knowing KDACIs 
targets is important for interpreting the effects of inhibitors both in research and clinical settings. We provide a detailed comparison of KDACI specificities at the level of affected sites, shedding insights into their specificities for acetylation sites in cells (Supplemental Figure 14; Supplemental note 2). For several KDACIs, the number of acetylation sites affected is not proportional to the number of KDACs they were found to inhibit in cell-free assays, which is consistent with recent studies demonstrating unexpected selectivities of KDACIs in-vitro ${ }^{18,19}$. Our results complement data from cell-free systems and emphasize the importance of evaluating KDACI effects at the acetylation site level to better understand and refine the specificities of KDACIs.

Analysis of acetylation in several genetically defined cells showed that broad-range inhibitors NAM, tubacin and bufexamac increase acetylation through inhibition of SIRT1 and HDAC6. Most other inhibitors of $\mathrm{Zn}^{2+}$-dependent deacetylases targeted multiple deacetylases (Fig. 1a) and commonly inhibit class I deacetylases. HDACs 1-3 are essential for cell viability, so we could not confirm their acetylation targets in knockout cells. However, several data indicate that these KDACIs regulate acetylation by specifically inhibiting class I HDACs: (i) acetylation was regulated in a site-specific manner, (ii) KDACIs increased acetylation of nuclear proteins, where their target deacetylases are present, and (iii) they increased acetylation of sites present on the N-terminal tails of histones, many of which are known to be regulated by class I deacetylases. Contrary to their reported broad inhibitory effects on deacetylases in cell-free systems ${ }^{18,41}$, most of these KDACIs increased acetylation of small but specific, subsets of the acetylome. The scope of these inhibitors was more limited than tubacin and bufexamac which inhibit only HDAC6 (and perhaps HDAC10), indicating that at concentrations applied here these inhibitors more potently inhibit class I deacetylases, but less potently inhibit HDAC6. The acetylation signatures of sirtuin inhibitors tenovin-6, NAM and sirtinol are not fully consistent with their predicted inhibitory profiles, indicating that disparitites between cell-free and cell-based acetylation profiles are not uncommon.

NAM and sirtinol were identified as inhibitors of yeast $\operatorname{Sir} 2^{33}$, and are used as sirtuin class deacetylase inhibitors. In this study, NAM-upregulated $12 \%$ of acetylation sites, whereas sirtinol affected less than $2 \%$ of sites, indicating that the scope of these inhibitors is vastly different. In contrast to several canonical KDACIs that inhibit $\mathrm{Zn}^{2+}$-dependent deacetylases, NAM increased acetylation of many non-histone proteins in the nucleus without causing widespread increase in histone acetylation levels. However, NAM increased acetylation on several histone-modifying enzymes such as acetyltransferases and methyltransferases, and other chromatin-associated proteins. The low overlap $(\sim 10 \%)$ between sites upregulated by NAM and all non-sirtuin targeting KDACIs suggests a minimal redundancy between sirtuins and $\mathrm{Zn}^{2+}$-dependent histone deacetylases. We found 
that most of the NAM-induced acetylation changes were mediated through inhibition of SIRT1, highlighting the broad target range of SIRT1 and suggesting that the physiological effects of NAM may be primarily mediated by inhibiting SIRT1 function.

Mitochondria exclusively contain $\mathrm{NAD}^{+}$-dependent deacetylases, and thus our results showing only a modest increase in mitochondrial acetylation by NAM are unexpected. The possible reasons for this remain unclear, but it could be that mitochondrial sirtuins are less sensitive to NAM, that NAM is excluded from mitochondria, or that NAM is rapidly metabolized to $\mathrm{NAD}^{+}$by nicotinamide phosphoribosyltransferase (NAMPT) ${ }^{42}$. Metabolic conversion of NAM to NAD ${ }^{+}$may result in lower effective concentrations of NAM, and subsequent import of $\mathrm{NAD}^{+}$into mitochondria may counteract its effect by activating sirtuins in this compartment. Tenovin-6 inhibits SIRT1 and SIRT2 in cell-free systems, whereas it weakly inhibits SIRT $3^{43}$; however, in cells, it increased acetylation of mitochondrial proteins, which suggests that it may increase acetylation by a yet unknown mechanism. With the emerging role of mitochondrial acetylation in regulating diverse metabolic pathways $^{44,45}$ and its relevance to the stress response ${ }^{46,47}$, the identification of tenovin- 6 as a potent inducer of mitochondrial acetylation may further facilitate studying the functional relevance of acetylation in this context.

Bufexamac has long been used as non-steroidal anti-inflammatory drug (NSAID) for the treatment of dermatoses. It was revoked in 2010 due to its adverse reactions that caused contact dermatitis ${ }^{39}$, but the mechanisms of bufexamac-mediated inflammation remained unknown. We showed that at higher concentrations $(>200 \mu \mathrm{M})$ it chelates cellular iron, potently stabilizes HIF1- $\alpha$, and induces downstream hypoxia-like responses (Supplemental Fig. 13). The concentrations of bufexamac present in most anti-inflammatory would be more than adequate to cause local hypoxia-like responses at the applied regions. Furthermore, the drug was applied at already inflamed regions, making it plausible that even much lower doses than that present in the prescribed ointments would have been sufficient to induce hypoxic responses. The connection between hypoxia and inflammation is well described ${ }^{48}$. Thus, our data provide new insights into the possible mechanistic basis of the bufexamac-inflammation paradox and illustrate the usefulness of unbiased proteomic analyses in discovering cellular mechanisms of drugs.

Although the global acetylome analyses applied here provide a powerful approach for obtaining an unbiased (non-hypothesis driven) picture of cellular targets of KDACIs, caution should be taken in interpreting the biological functions of KDACI-regulated acetylation sites. First, while we have applied a 2-fold change as a cut-off to classify KDACI-regulated sites, it is possible that less robust 
changes (i.e. $50 \%$ increases in acetylation) could have important biological consequences. Second, in this work we compared changes in acetylation using a single inhibitor dose and time point, though it is plausible that the effects of KDACIs are dose- and time-dependent, and that by varying doses and treatment times one may identify additional substrates. Third, our analyses cannot distinguish between direct and indirect targets of deacetylases inhibited by KDACIs. Thus, for functional investigations of specific acetylation sites it is important to independently verify their regulation by KDACIs using varying doses and time points. Nevertheless, our results offer a snapshot of the KDACI substrate spectrum at the most commonly used doses, providing a road-map for further investigations.

In summary, our results portray a global picture of acetylation sites regulated by KDACIs and their several downstream deacetylases, and provide a detailed comparative view of their regulatory scope.These results exemplify the usefulness of quantitative MS screens in revealing endogenous acetylation profiles for known KDACIs in cells and in identifying selective KDACIs for sites of interest. In addition to elucidating broad outlines of acetylation inhibition, we anticipate that this resource dataset will be useful in investigating the functional roles of deacetylases and their target sites, in understanding the molecular basis of drugs targeting deacetylases, in evaluating specificity of novel KDACIs, and in developing new therapies.

\section{Footnotes}

The supplementary information contains part of the data reported in this manuscript and a detailed description of all materials and methods used to obtain the data.

\section{Acknowledgements}

We thank the members of the department of proteomics at CPR for their helpful discussions. We thank Takeo Narita for providing help with bioinformatic analyses. We thank Richard Lavallee, Dorte Bekker-Jensen, Heidi Cordula Grell, and Billy Smith for their technical support. We gratefully acknowledge Drs. Michael W. McBurney for providing SIRT1 knockcout MEFs, Danny Reinberg for providing SIRT2 knockout MEFs, and Raul Mostoslavsky for providing SIRT6 knockout MEFs. This work was supported by the Hallas Møller Investigator grant from the Novo Nordisk Foundation to CC. SAW and PB were supported by individual postdoctoral grants from the Danish Research Council (FSS: 10-085134, FSS: 12-12610). CC is supported by the EMBO Young Investigator program. The Center for Protein Research is supported by a generous grant from the Novo Nordisk 
Foundation (Grant no. NNF14CC0001). JEB is supported by a grant from the Doris Duke Charitable Foundation.

\section{Author contributions}

CS performed most of the experiments and collected data. BTW performed initial experiments, and obtained data in MV4-11 cells, SAW helped with bioinformatic analyses, PB assisted with immunofluorescence microscopy, MY provided HDAC6 knockout cells, LJJ performed average linkage clustering analysis of KDACIs, WS performed UV/VIS spectroscopy, JQ synthesized JQ12 and performed in-vitro KDAC enzymatic assays for this compound, ARM, NJW and SL provided tenovin-6, JC helped with computational analysis of mass spectrometry data, PM provided critical research reagents, $\mathrm{MM}$ was involved in the planning of the tenovin- 6 experiments and provided infrastructure for initial test experiments, JEB designed experiments, provided pandacostat and JQ12, CC planned the project, $\mathrm{CC}$ and CS wrote the manuscript; all co-authors provided input for writing the paper.

\section{Declaration of conflict of interest}

None of the authors have a conflict of interest.

\section{MS data availability}

The mass spectrometry proteomics data have been deposited to the ProteomeXchange Consortium (http://proteomecentral.proteomexchange.org) via the PRIDE partner repository ${ }^{49}$ with the dataset identifier PXD001377. 


\section{Online Methods}

\section{Materials}

KDACIs were purchased either from Sigma-Aldrich, Selleckchem or Chemie-Tek. All other standard chemicals were obtained from Sigma-Aldrich and Merck KGaA. Cell culture media and reagents were from Invitrogen. SILAC amino acids were purchased from Cambridge Isotope Laboratories. Agarose-conjugated anti-acetyllysine antibodies were purchased from Immunechem Inc. (Catalog no.: ICP0388). Antibodies for immuno-blotting were purchased from Abcam (H3AcK122, ab33309), Millipore (H4Ac-K16, 07-329), Cell Signalling (Ac-tubulin, 5335P; Ac-Lysine, 9441; SIRT1, 2028; c-myc, 2276), Sigma Aldrich (vinculin, V9264; tubulin, T5326), Santa Cruz (SMC3, sc-376352; HDAC8, sc-374180), and Epitomics (HIF1- $\alpha, 2015-1)$. Anti-mHDAC6 antibody was generated by $\mathrm{P}$. Matthias lab; purified recombinant protein antigen corresponding to the Cterminal ZnF-domain of mHDAC6 was injected into rabbits (Pocono rabbit farm \& laboratory; USA) and serum containing mHDAC6 antibody was used for further antibody purification.

\section{HDAC biochemical assay for JQ12}

JQ12 was tested against HDAC1-9 and the activity was determined with an optimized homogenous assay performed in a 384-well plate. Compound was incubated with HDAC enzyme for $3 \mathrm{~h}$. Reactions were performed in assay buffer (50 mM HEPES, $100 \mathrm{mM} \mathrm{KCl,} \mathrm{0.001 \%} \mathrm{Tween-20,} 0.05 \%$ BSA and pH 7.4. Additional $200 \mu \mathrm{M}$ TCEP was added for HDAC6) and followed by fluorogenic release of 7-amino-4-methylcoumarin from substrate upon deacetylase and trypsin enzymatic activity. Fluorescence measurements were obtained every five minutes using a multilabel plate reader and plate-stacker (Envision; Perkin-Elmer). Each plate was analyzed by plate repeat, and the first derivative within the linear range was imported into analytical software (Spotfire DecisionSite). Replicate experimental data from incubations with inhibitor were normalized to DMSO controls ([DMSO] $<0.5 \%)$. IC50 was determined by logistic regression with unconstrained maximum and minimum values (Supplemental Figures 1B and 1C). The recombinant, full-length HDAC protein (BPS Biosciences) was incubated with fluorophore conjugates substrate, MAZ1600 and MAZ1675 at $\mathrm{Km}=$ [substrate].

\section{Cell culture, SILAC labeling, preparation of cell lysates and protein digestion}

Double SILAC Experiments: 
HeLa (ATCC: CCL-2) and MV4-11 (ATCC: CRL-9591) cells, were grown in DMEM and RPMI, respectively, supplemented with $10 \%$ FBS, 2 mM L-glutamine, and $1 \%$ penicillin/streptomycin. For SILAC labeling, cells were cultured in the appropriate medium containing either L-arginine and Llysine (light label) or L-arginine- ${ }^{13} \mathrm{C}_{6}{ }^{-15} \mathrm{~N}_{4}$ and L-lysine- $-{ }^{13} \mathrm{C}_{6}{ }^{15} \mathrm{~N}_{2}$ (heavy label). All cells were cultured at $37^{\circ} \mathrm{C}$ in a humidified incubator at $5 \% \mathrm{CO}_{2}$. At a confluency of $\sim 90 \%$, heavy-labeled cells were treated for $16 \mathrm{~h}$ with the respective HDAC inhibitor (for concentrations used in this study, please refer to Supplemental Table 1), whereas light labeled cells were mock-treated with DMSO and $\mathrm{H}_{2} \mathrm{O}$, respectively. Each experiment was performed at least in biological duplicates. Cells were washed twice with PBS and lysed in modified RIPA buffer (50 mM Tris- $\mathrm{HCl}$ (pH 7.5), $150 \mathrm{mM}$ $\mathrm{NaCl}, 1 \mathrm{mM}$ EDTA, $1 \% \mathrm{NP}-40$, and $0.1 \%$ sodium deoxycholate, supplemented with complete protease inhibitor mix (Roche). Lysates were mixed with $1 / 10$ volume of $5 \mathrm{M} \mathrm{NaCl}$ to release chromatin-bound proteins and incubated for $15 \mathrm{~min}$ on ice. Subsequently, lysates were homogenized by sonication $(6 \times 10 \mathrm{sec}, 15 \mathrm{~W})$, cleared by centrifugation $\left(20.000 \times \mathrm{g}, 15 \mathrm{~min}, 4^{\circ} \mathrm{C}\right)$, and proteins were precipitated overnight at $-20^{\circ} \mathrm{C}$ by the addition of 4 volumes of ice-cold acetone. Precipitates were redissolved in $8 \mathrm{M}$ urea (6 $\mathrm{M}$ urea, $2 \mathrm{M}$ thiourea) and protein concentration was determined by Quick-start Bradford assay (Bio-Rad). Corresponding SILAC labeled proteins (heavy and light labeled) were mixed in a 1:1 ratio, reduced with $1 \mathrm{mM}$ DTT (45 min, RT), and alkylated by $5.5 \mathrm{mM}$ chloracetamide (45 min, RT). Following, $20 \mathrm{mg}$ proteins were digested by endoproteinase Lys-C (1:100 w/w; Wako) and, after 4-fold dilution with HEPES buffer $(50 \mathrm{mM}$; pH 7.5), by modified sequencing grade trypsin (1:100 w/w; Sigma-Aldrich). Digestion was stopped by the addition of trifluoroacetic acid (TFA) to a final concentration of $1 \%$.

Triple SILAC Experiments:

Mouse embryonic fibroblast (MEF) cells (wildtype (WT), Hdac6 ${ }_{4}^{-1-50}, \operatorname{Sirt}^{-/-51}, \mathrm{Sirt}^{-/-} 52, \mathrm{Sirt}^{-/-}$. 33 ) were grown in DMEM as described above. For SILAC labeling, WT cells were cultured in DMEM containing either L-arginine and L-lysine (light label), or in medium supplemented with L-arginine- ${ }^{13} \mathrm{C}_{6}$ and L-lysine- ${ }^{2} \mathrm{H}_{4}$ (medium label), whereas the corresponding knockout (KO) cells were heavy labeled using DMEM containing L-arginine- ${ }^{13} \mathrm{C}_{6^{-}}{ }^{15} \mathrm{~N}_{4}$ and L-lysine- ${ }^{13} \mathrm{C}_{6^{-}}{ }^{15} \mathrm{~N}_{2}$. At a confluency of $\sim 90 \%$, medium-labeled WT cells were treated for $16 \mathrm{~h}$ with the respective HDACIs, whereas light labeled WT, and heavy labeled KO cells were mock-treated with DMSO and $\mathrm{H}_{2} \mathrm{O}$, respectively. All experiments were performed in at least two biological replicates. Cells were harvested as described above, lysed, and proteins were mixed in a 1:1:1 ratio prior to reduction with DTT, alkylation with chloracetamide and trypsin digestion, as described above. 


\section{Purification of peptides and enrichment of acetylated peptides}

Digested protein samples were cleared for precipitates by centrifugation $(2,500 \mathrm{x} \mathrm{g}, 5 \mathrm{~min})$ and loaded onto reversed-phase C18 Sep-Pak columns (Waters), pre-equilibrated with $5 \mathrm{ml}$ acetonitrile and $2 \times 5 \mathrm{ml} 0.1 \%$ TFA. Peptides were washed with $0.1 \%$ TFA and $\mathrm{H}_{2} \mathrm{O}$, eluted with $50 \%$ acetonitrile (ACN) and mixed with $100 \mu \mathrm{l}$ 10x IAP buffer (500 mM MOPS; pH 7.2, $100 \mathrm{mM} \mathrm{Na-}$ phosphate, $500 \mathrm{mM} \mathrm{NaCl}$ ). Subsequently, ACN was removed and volume of samples was adjusted to $1 \mathrm{ml}$ by $\mathrm{H}_{2} \mathrm{O}$. For proteome analysis, small aliquots of total peptides were fractionated using micro-SCX columns in a stage-tip format ${ }^{54}$. Peptide fractions were purified and concentrated with reversed-phase StageTips as described ${ }^{54}$. For acetylation enrichment, modified peptides were immunoenriched using anti-acetyllysine antibodies $(40 \mu \mathrm{l} / \mathrm{IP})$ as described previously ${ }^{22}$. Enriched peptides were fractionated and purified as described for proteome samples. Peptides were eluted with $40 \mu \mathrm{l}$ of buffer B (60\% ACN, $0.1 \%$ TFA) and organic solvent was removed in a SpeedVac concentrator. The final sample volume was adjusted to $7 \mu 1$ with buffer $A^{*}(0.5 \%$ acetic acid, $0.2 \%$ TFA).

\section{Mass spectrometric analysis}

Peptide fractions were analyzed by online nanoflow LC-MS/MS with a Proxeon easy nLC system (Thermo) connected to a Q Exactive mass spectrometer (Thermo) as described previously ${ }^{55}$. Briefly, peptide samples were loaded onto C18 reversed-phase chromatography columns (length $15 \mathrm{~cm}$, inner diameter $75 \mu \mathrm{m}$ ) and eluted with a linear gradient of 6-40\% $\mathrm{ACN} / \mathrm{H}_{2} \mathrm{O}$ containing $0.5 \%$ acetic acid. Eluted peptides were ionized by electrospray-ionization and measured in the mass spectrometer. Typical mass spectrometric conditions were: spray voltage: $2.0 \mathrm{kV}$, no sheath and auxiliary gas flow, heated capillary temperature: $275^{\circ} \mathrm{C}$. The Q Exactive under Xcalibur 2.2 with LTQ Orbitrap Tune Plus Developers Kit version 2.6.0.1042 software was operated in data dependent mode to automatically switch between MS and MS2 acquisitions as described previously ${ }^{56}$.

\section{Peptide identification and quantification}

All MS data were analyzed with MaxQuant (development version 1.2.7.1) ${ }^{57}$. All SILAC pairs were quantified and MS/MS spectra were searched against the human Uniprot FASTA database (released in February 2012) to identify corresponding proteins. The false-discovery rate (FDR) was fixed to a threshold of $1 \%$ FDR at peptide and protein level and all peptide identifications were filtered for length and mass error. FDR was estimated using a target-decoy database search approach ${ }^{58}$. 
Cysteine carbamidomethylation was searched as a fixed modification, whereas methionine oxidation, $\mathrm{N}$-acetyl protein, and acetylation of lysine were chosen as variable modifications.

\section{Immunofluorescence microscopy}

HeLa cells were treated without or with bufexamac $(50$ and $250 \mu \mathrm{M})$ for $4 \mathrm{~h}$ at $37^{\circ} \mathrm{C}$. Subsequently, cells were fixed by $2 \%$ paraformaldehyde (PFA) in PBS and permeabilized with $0.2 \%$ Triton-X in PBS. After blocking with $5 \%$ bovine serum albumin (BSA) in PBS, cells were incubated with primary antibody (anti-acetyl-tubulin and anti-HIF1- $\alpha$ ) and subsequently with anti-rabbit secondary antibody coupled to Alexa Fluor 488 and 568 (Molecular Probes). Cells were mounted by using DAPI containing Vectashield mounting medium (Vector labs). Confocal images were acquired on a LSM-780 (Carl Zeiss Microimaging Inc.) mounted on Zeiss-Axiovert 100M equipped with PlanNeofluar 40 x/1.3 oil immersion objective.

\section{Luciferase assays}

HeLa cells were transfected with hypoxia responsive element (HRE)-Luciferase plasmid (Addgene; plasmid No. $26731 ;^{59}$ ) and treated as indicated. Activation of hypoxia response elements was determined by measuring luciferase activity using the Dual-Glo Luciferase Kit (Promega) according to the manufacturer's protocol.

\section{QPCR}

HeLa cells were pretreated without or with bufexamac (50 and $250 \mu \mathrm{M})$ and Desferoxamine mesylate $(200 \mu \mathrm{M})$, and subsequently RNA was isolated using the Qiagen RNeasy kit (Qiagen) according to manufacturer's protocol. The same procedure was used to obtain RNA from wt and KO MEF cells. Total RNA was quantified using a nano-drop spectrophotometer (Thermo) and complementary DNA (cDNA) was synthesized using the QuantiTect Rev. Transcription kit (Qiagen). Reverse transcription reactions were diluted $1: 5$ with $\mathrm{H}_{2} \mathrm{O}$ and stored at $-20^{\circ} \mathrm{C}$. Quantitative PCR was performed using a Stratagene Mx3005P instrument. Experimental data was analyzed with MxPro software. Sample setup and QPCR reactions were performed as described ${ }^{60}$. For each QPCR reaction run a glyceraldehyde-3- phosphate dehydrogenase (Gapdh) standard curve was prepared by 1,000 -fold serial dilution (10x at each point) and the PCR efficiency was determined. The PCR efficiency was then used to calculate the abundance of cDNA for the target gene relative to Gapdh. The following primers (Hs, Homo sapiens; Mm, Mus musculus) were used for QPCR: HsGapdh, forward: 5'-CAGCGACACCCACTCCTCCA-3' and reverse: 5'GCTGGTGGTCCAGGGGTCTT-3'; HsVegfA, forward: 5'-CGAGACTCCGGCGGAAGCAT-3' 
and reverse: 5'-CGGCCGCGGTGTGTCTACAG-3'; MmGapdh, forward: 5'TCCATGACAACTTTGGCATTG-3' and reverse: 5'-CAGTCTTCTGGGTGGCAGTGA-3'; MmSirt2, forward: 5'-CGAAGGAGTGACACGCTACATG-3' and reverse: 5'GGTGGTACTTCTCCAGGTTTGC-3'; MmSirt6, forward: 5'CAGTACGTCAGAGACACGGTTG-3' and reverse: 5'- GTCCAGAATGGTGTCTCTCAGC-3'.

\section{Immuno-blotting}

For immuno-blotting, protein lysates were prepared in modified RIPA buffer and protein amounts measured by Bradford assay. Equal amounts of proteins were precipitated by ice-cold acetone and precipated proteins were recovered by centrifugation $\left(20.000 \mathrm{x} \mathrm{g}, 2 \mathrm{~min}, 4^{\circ} \mathrm{C}\right)$. Proteins were redissolved in 4x sample buffer containing 100 mM DTT, and separated on NuPAGE Novex 4-12\% Bis-Tris gels (Invitrogen) and visualized by immuno-staining with the indicated antibodies.

\section{Immuno-precipitation of SMC3}

HeLa cells were treated with different concentrations of PCI34501, washed with PBS, lysed with IPwash buffer $\left(5 \mathrm{mM} \mathrm{MgCl}, 1 \mathrm{mM} \mathrm{CaCl}_{2}, 1 \mathrm{mM}\right.$ EDTA, $0.1 \%$ Triton X-100 in PBS; pH 7.4, supplemented with complete protease inhibitor mix (Roche)) containing $1 \%$ Triton X-100 and clearified by centrifugation $\left(20.000 \mathrm{x} \mathrm{g}, 15 \mathrm{~min}, 4^{\circ} \mathrm{C}\right)$ ). For detection of HDAC8-inhibitor dependent acetylation of SMC3, protein G magnetic Dynabeads (Dynal) were pre-loaded with anti-SMC3 antibody for $2 \mathrm{~h}$ at $4{ }^{\circ} \mathrm{C}$. Control IPs were performed using an antibody against c-Myc epitope tag. After coating, beads were washed three times with ice-cold IP-wash buffer. Subsequently, beads were incubated for $2 \mathrm{~h}$ at $4^{\circ} \mathrm{C}$ with cell lysates. After washing three times with IP-wash buffer and three times with cold PBS proteins were eluted with $50 \mu \mathrm{l}$ SDS sample buffer for $10 \mathrm{~min}$ at $65^{\circ} \mathrm{C}$. Eluates were analyzed by SDS-PAGE and subsequent immuno-blotting using a pan-anti-acetyllysine antibody.

\section{Determination of cell death}

Human promyelocytic leukemia (HL-60) cells (ATCC: CCL-240) were treated as indicated. Subsequently, cell viability was determined using propidium iodide (PI) staining solution (Cayman Chemical Company). Cells were stained according to the manufacturer's protocol and PI fluorescence was measured in a plate reader.

\section{Iron binding assay}


Bufexamac ( $5 \mathrm{mM}$ in methanol) and desferoxamine mesylate ( $5 \mathrm{mM}$ in methanol), were titrated in 2 $\mu \mathrm{l}$ increments into $500 \mu \mathrm{l}$ of a $100 \mu \mathrm{M}$ methanolic $\mathrm{FeCl}_{3}$ solution and iron-complex formation was measured at a wavelength of $490 \mathrm{~nm}$ using a Varian Cary 50Bio UV-Vis Spectrophotometer. Absorption was plotted against chelator concentration and fitted to a Langmuir (1:1) isotherm $\left[y=a * \frac{x}{K_{D}+x}\right]$ to derive the dissociation constant $\left(\mathrm{K}_{\mathrm{D}}\right)$. All binding assays were performed using methanol as solvent due to very low solubility of bufexamac in aqueous solutions, and thus, these $K_{D}$ values may differ from values in aqueous solutions.

\section{Data analysis}

Statistical analysis was performed using the $\mathrm{R}$ software environment (http:// http://www.rproject.org/) and biochemical data was analyzed using Graphpad Prism (version 5). Gene ontology (GO) term enrichment was calculated using Fisher exact test. $P$-values were adjusted for multiple hypotheses testing using the false discovery rate (FDR)-method. Protein interaction network analysis was performed using interaction data from the STRING database (version 9) ${ }_{4}^{61}$. Only interactions with a STRING score above 0.7 are represented in the network, and visualized using Cytoscape ${ }^{62}$ and ClueGO / GOlorize plug-ins ${ }_{4}^{63,64}$.

\section{Site-based clustering of inhibitors}

To define an acetylation site-based similarity of two inhibitors, we selected the sites that were identified for both KDACIs and showed increased acetylation (SILAC ratio >2) for at least one of the two inhibitors. We next log-transformed the ratios and defined the similarity as the Pearson correlation. We used this definition to calculate an all-against-all similarity matrix for the 19 inhibitors. Based on this similarity matrix, we performed average linkage clustering using the using the OC software (http://www.compbio.dundee.ac.uk/downloads/oc/). Finally, we visualized the resulting cluster tree as an unrooted tree using iTOL (http://itol.embl.de/).

\section{References}

1 Grunstein, M. Histone acetylation in chromatin structure and transcription. Nature 389, 349352 (1997).

2 Cheung, W. L., Briggs, S. D. \& Allis, C. D. Acetylation and chromosomal functions. Current opinion in cell biology 12, 326-333 (2000).

3 Valenzuela-Fernandez, A., Cabrero, J. R., Serrador, J. M. \& Sanchez-Madrid, F. HDAC6: a key regulator of cytoskeleton, cell migration and cell-cell interactions. Trends in cell biology 18, 291-297 (2008). 
4 Yang, X. J. \& Seto, E. Lysine acetylation: codified crosstalk with other posttranslational modifications. Molecular cell 31, 449-461 (2008).

5 Haberland, M., Montgomery, R. L. \& Olson, E. N. The many roles of histone deacetylases in development and physiology: implications for disease and therapy. Nature reviews 10, 32-42 (2009).

6 Finkel, T., Deng, C. X. \& Mostoslavsky, R. Recent progress in the biology and physiology of sirtuins. Nature 460, 587-591 (2009).

7 Montgomery, R. L., Hsieh, J., Barbosa, A. C., Richardson, J. A. \& Olson, E. N. Histone deacetylases 1 and 2 control the progression of neural precursors to neurons during brain development. Proceedings of the National Academy of Sciences of the United States of America 106, 7876-7881, doi:10.1073/pnas.0902750106 (2009).

8 Bolden, J. E., Peart, M. J. \& Johnstone, R. W. Anticancer activities of histone deacetylase inhibitors. Nature reviews. Drug discovery 5, 769-784, doi:10.1038/nrd2133 (2006).

9 Marks, P. A. The clinical development of histone deacetylase inhibitors as targeted anticancer drugs. Expert opinion on investigational drugs 19, 1049-1066 (2010).

10 Archin, N. M. et al. Administration of vorinostat disrupts HIV-1 latency in patients on antiretroviral therapy. Nature 487, 482-485 (2012).

11 Grabiec, A. M., Tak, P. P. \& Reedquist, K. A. Function of histone deacetylase inhibitors in inflammation. Critical reviews in immunology 31, 233-263 (2011).

12 Kazantsev, A. G. \& Thompson, L. M. Therapeutic application of histone deacetylase inhibitors for central nervous system disorders. Nature reviews. Drug discovery 7, 854-868 (2008).

13 Iyer, A., Fairlie, D. P. \& Brown, L. Lysine acetylation in obesity, diabetes and metabolic disease. Immunology and cell biology 90, 39-46 (2012).

14 Khan, O. \& La Thangue, N. B. HDAC inhibitors in cancer biology: emerging mechanisms and clinical applications. Immunol Cell Biol 90, 85-94, doi:10.1038/icb.2011.100 (2012). $\mathrm{Xu}$, W. S., Parmigiani, R. B. \& Marks, P. A. Histone deacetylase inhibitors: molecular mechanisms of action. Oncogene 26, 5541-5552 (2007).

16 Tan, J., Cang, S., Ma, Y., Petrillo, R. L. \& Liu, D. Novel histone deacetylase inhibitors in clinical trials as anti-cancer agents. Journal of hematology \& oncology 3, 5 (2010). Dell'Aversana, C., Lepore, I. \& Altucci, L. HDAC modulation and cell death in the clinic. Experimental cell research 318, 1229-1244, doi:10.1016/j.yexcr.2012.01.025 (2012).

18 Bradner, J. E. et al. Chemical phylogenetics of histone deacetylases. Nature chemical biology 6, 238-243, doi:nchembio.313 [pii]

10.1038/nchembio.313 (2010).

19 Bantscheff, M. et al. Chemoproteomics profiling of HDAC inhibitors reveals selective targeting of HDAC complexes. Nature biotechnology 29, 255-265, doi:nbt.1759 [pii]

10.1038/nbt.1759 (2012).

20 Salisbury, C. M. \& Cravatt, B. F. Activity-based probes for proteomic profiling of histone deacetylase complexes. Proc Natl Acad Sci U S A 104, 1171-1176 (2007).

21 Ong, S. E. et al. Stable isotope labeling by amino acids in cell culture, SILAC, as a simple and accurate approach to expression proteomics. Mol Cell Proteomics 1, 376-386 (2002).

22 Choudhary, C. et al. Lysine acetylation targets protein complexes and co-regulates major cellular functions. Science 325, 834-840 (2009).

23 Weinert, B. T. et al. Lysine succinylation is a frequently occurring modification in prokaryotes and eukaryotes and extensively overlaps with acetylation. Cell reports 4, 842 851, doi:10.1016/j.celrep.2013.07.024 (2013).

24 Sterner, D. E. \& Berger, S. L. Acetylation of histones and transcription-related factors. Microbiol Mol Biol Rev 64, 435-459 (2000). 
Drogaris, P. et al. Histone deacetylase inhibitors globally enhance $\mathrm{h} 3 / \mathrm{h} 4$ tail acetylation without affecting h3 lysine 56 acetylation. Sci Rep 2, 220, doi:10.1038/srep00220 (2012). Buggy, J. J. et al. CRA-024781: a novel synthetic inhibitor of histone deacetylase enzymes with antitumor activity in vitro and in vivo. Molecular cancer therapeutics 5, 1309-1317, doi:10.1158/1535-7163.MCT-05-0442 (2006).

27 Haggarty, S. J., Koeller, K. M., Wong, J. C., Grozinger, C. M. \& Schreiber, S. L. Domainselective small-molecule inhibitor of histone deacetylase 6 (HDAC6)-mediated tubulin deacetylation. Proceedings of the National Academy of Sciences of the United States of America 100, 4389-4394, doi:10.1073/pnas.0430973100 (2003).

28 Balasubramanian, S. et al. A novel histone deacetylase 8 (HDAC8)-specific inhibitor PCI34051 induces apoptosis in T-cell lymphomas. Leukemia 22, 1026-1034 (2008).

29 Rolef Ben-Shahar, T. et al. Eco1-dependent cohesin acetylation during establishment of sister chromatid cohesion. Science 321, 563-566 (2008).

30 Unal, E. et al. A molecular determinant for the establishment of sister chromatid cohesion. Science 321, 566-569 (2008).

31 Zhang, J. et al. Acetylation of Smc3 by Eco1 is required for S phase sister chromatid cohesion in both human and yeast. Molecular cell 31, 143-151 (2008).

32 Deardorff, M. A. et al. HDAC8 mutations in Cornelia de Lange syndrome affect the cohesin acetylation cycle. Nature (2012).

33 Bitterman, K. J., Anderson, R. M., Cohen, H. Y., Latorre-Esteves, M. \& Sinclair, D. A. Inhibition of silencing and accelerated aging by nicotinamide, a putative negative regulator of yeast sir2 and human SIRT1. J Biol Chem 277, 45099-45107, doi:10.1074/jbc.M205670200

M205670200 [pii] (2002).

34 Chen, Y. et al. Quantitative acetylome analysis reveals the roles of SIRT1 in regulating diverse substrates and cellular pathways. Molecular \& cellular proteomics : MCP 11, 10481062, doi:10.1074/mcp.M112.019547 (2012).

35 Kim, S. C. et al. Substrate and functional diversity of lysine acetylation revealed by a proteomics survey. Molecular cell 23, 607-618, doi:10.1016/j.molcel.2006.06.026 (2006).

36 Zhao, S. et al. Regulation of cellular metabolism by protein lysine acetylation. Science 327, 1000-1004 (2010).

37 Zhang, X. et al. HDAC6 modulates cell motility by altering the acetylation level of cortactin. Molecular cell 27, 197-213 (2007).

38 Uter, W. \& Schnuch, A. EMA revokes marketing authorization for bufexamac. Contact dermatitis 64, 235-236 (2011).

39 Fukuda, H., Sato, Y., Usami, N., Yokouchi, Y. \& Mukai, H. Contact dermatitis caused by bufexamac sparing the eruption of herpes zoster. J Dermatol 39, 405-407 (2012).

40 Jeong, J. W. et al. Regulation and destabilization of HIF-1alpha by ARD1-mediated acetylation. Cell 111, 709-720 (2002).

41 Dokmanovic, M., Clarke, C. \& Marks, P. A. Histone deacetylase inhibitors: overview and perspectives. Molecular cancer research : MCR 5, 981-989, doi:10.1158/1541-7786.MCR07-0324 (2007).

42 Yang, H. et al. Nutrient-sensitive mitochondrial NAD+ levels dictate cell survival. Cell 130, 1095-1107 (2007).

43 Lain, S. et al. Discovery, in vivo activity, and mechanism of action of a small-molecule p53 activator. Cancer cell 13, 454-463, doi:10.1016/j.ccr.2008.03.004 (2008).

44 Anderson, K. A. \& Hirschey, M. D. Mitochondrial protein acetylation regulates metabolism. Essays Biochem 52, 23-35, doi:10.1042/bse0520023 (2012). 
45 Hirschey, M. D., Shimazu, T., Huang, J. Y., Schwer, B. \& Verdin, E. SIRT3 regulates mitochondrial protein acetylation and intermediary metabolism. Cold Spring Harbor symposia on quantitative biology 76, 267-277, doi:10.1101/sqb.2011.76.010850 (2011).

46 Wagner, G. R. \& Payne, R. M. Mitochondrial acetylation and diseases of aging. J Aging Res 2011, 234875, doi:10.4061/2011/234875 (2011).

47 He, W., Newman, J. C., Wang, M. Z., Ho, L. \& Verdin, E. Mitochondrial sirtuins: regulators of protein acylation and metabolism. Trends Endocrinol Metab 23, 467-476, doi:10.1016/j.tem.2012.07.004 (2012). Eltzschig, H. K. \& Carmeliet, P. Hypoxia and inflammation. N Engl J Med 364, 656-665, doi:10.1056/NEJMra0910283 (2011).

49 Vizcaino, J. A. et al. ProteomeXchange provides globally coordinated proteomics data submission and dissemination. Nature biotechnology 32, 223-226, doi:10.1038/nbt.2839 (2014).

50 Zhang, Y. et al. Mice lacking histone deacetylase 6 have hyperacetylated tubulin but are viable and develop normally. Molecular and cellular biology 28, 1688-1701, doi:10.1128/MCB.01154-06 (2008).

51 McBurney, M. W. et al. The mammalian SIR2alpha protein has a role in embryogenesis and gametogenesis. Molecular and cellular biology 23, 38-54 (2003).

52 Vaquero, A. et al. SirT2 is a histone deacetylase with preference for histone H4 Lys 16 during mitosis. Genes \& development 20, 1256-1261, doi:10.1101/gad.1412706 (2006).

53 Mostoslavsky, R. et al. Genomic instability and aging-like phenotype in the absence of mammalian SIRT6. Cell 124, 315-329, doi:10.1016/j.cell.2005.11.044 (2006).

54 Rappsilber, J., Mann, M. \& Ishihama, Y. Protocol for micro-purification, enrichment, prefractionation and storage of peptides for proteomics using StageTips. Nature protocols $\mathbf{2}$, 1896-1906, doi:10.1038/nprot.2007.261 (2007).

55 Michalski, A. et al. Mass spectrometry-based proteomics using Q Exactive, a highperformance benchtop quadrupole Orbitrap mass spectrometer. Mol Cell Proteomics 10, M111 011015, doi:10.1074/mcp.M111.011015 (2011).

56 Kelstrup, C. D., Young, C., Lavallee, R., Nielsen, M. L. \& Olsen, J. V. Optimized Fast and Sensitive Acquisition Methods for Shotgun Proteomics on a Quadrupole Orbitrap Mass Spectrometer. Journal of proteome research, doi:10.1021/pr3000249 (2012).

57 Waanders, L. F. et al. A novel chromatographic method allows on-line reanalysis of the proteome. Molecular \& cellular proteomics : MCP 7, 1452-1459, doi:10.1074/mcp.M800141-MCP200 (2008).

58 Elias, J. E. \& Gygi, S. P. Target-decoy search strategy for increased confidence in large-scale protein identifications by mass spectrometry. Nat Methods 4, 207-214 (2007).

59 Emerling, B. M., Weinberg, F., Liu, J. L., Mak, T. W. \& Chandel, N. S. PTEN regulates p300-dependent hypoxia-inducible factor 1 transcriptional activity through Forkhead transcription factor 3a (FOXO3a). Proceedings of the National Academy of Sciences of the United States of America 105, 2622-2627, doi:10.1073/pnas.0706790105 (2008).

60 Weinert, B. T. et al. Real-time PCR analysis of genes encoding tumor antigens in esophageal tumors and a cancer vaccine. Cancer Immun 9, 9 (2009).

61 Szklarczyk, D. et al. The STRING database in 2011: functional interaction networks of proteins, globally integrated and scored. Nucleic acids research 39, D561-568, doi:10.1093/nar/gkq973 (2011).

62 Cline, M. S. et al. Integration of biological networks and gene expression data using Cytoscape. Nature protocols 2, 2366-2382, doi:10.1038/nprot.2007.324 (2007).

63 Bindea, G. et al. ClueGO: a Cytoscape plug-in to decipher functionally grouped gene ontology and pathway annotation networks. Bioinformatics 25, 1091-1093, doi:10.1093/bioinformatics/btp101 (2009). 
64 Garcia, O. et al. GOlorize: a Cytoscape plug-in for network visualization with Gene Ontology-based layout and coloring. Bioinformatics 23, 394-396,

doi:10.1093/bioinformatics/btl605 (2007).

65 Nagaraj, N. et al. Deep proteome and transcriptome mapping of a human cancer cell line. Molecular systems biology 7, 548, doi:10.1038/msb.2011.81 (2011). 


\section{Figure Legends}

Figure 1 | Quantitative profiling of the KDACI regulated acetylome. a, An overview of the 19 KDACIs used for cell-based acetylome analysis and their reported specificities for human deacetylases based on cell-free assays. KDACs shown in dark grey background have been shown to be expressed in HeLa cells $s^{65}$. b, Schematic representation of the experimental design. SILAC labeled HeLa cells were treated for $16 \mathrm{~h}$ with KDAC inhibitors or vehicle control. Subsequently, proteins were extracted and proteolysed using Lys-C and trypsin. Acetylated peptides were enriched with anti-acetyllysine antibodies and fractionated by strong-cation exchange (SCX) chromatography. Peptides were analyzed by mass spectrometry and data were used for downstream bioinformatic analyses. c, The figure shows the number of acetylation sites quantified and the fraction of acetylation sites regulated by individual KDACIs. The left part of the figure shows total number of quantified acetylation sites for each inhibitor. The bar chart shows the fraction of upregulated sites (>2-fold increase, shown in red), and the fraction of down-regulated sites ( $>2$-fold decrease, shown in blue). The numbers next to the bars indicate percent of up-or down-regulated sites, and the number of up- or down-downregulated sites for each KDACI is indicated within parenthesizes.

\section{Figure 2 | Specificity of KDACIs and subcellular distribution of KDACI-upregulated} acetylated proteins. a, Site-based specificity analysis of KDACIs in HeLa cells. SILAC ratios of KDACI upregulated sites were used to calculate pair-wise Pearson correlation coefficients for all KDACIs and the inhibitors were grouped based on their correlation values using average linkage clustering approach (see supplemental fig. 14 for pair-wise correlations). Node-size reflects number of upregulated sites, and line thickness corresponds to the degree of correlation. $\mathbf{b}$, Subcellular distribution of proteins with KDACI-upregulated acetylation sites. The bar plot shows the fraction of KDACI-upregulated acetylated proteins annotated with the indicated Gene Ontology cellular compartment (GOCC) terms. As a reference, the first bar indicates subcellular distribution of all acetylation sites identified in this study. The dendrogram at the top of the plot shows similarity of KDACIs for upregulated subcellular acetylomes. Sirtinol, JQ12 and PCI34051 were excluded from this analysis due to insufficient number of upregulated sites. c, Profile of KDACI-regulated histone acetylation sites. The heatmap shows quantified histone acetylation sites and their regulation by KDACIs. The dendrogram illustrates similarity of KDACIs for histone acetylation sites. Black matrix areas show sites not identified for an individual inhibitor. d, Overlap of acetylation sites upregulated in cells treated with broad-range KDACIs NAM, tenovin-6, tubacin, and PCI24781. The diagram displays the number of upregulated sites in response to each KDACI, as well as the number of sites found in two, three or all four of these inhibitors. 
Figure 3 | Nicotinamide increases acetylation of nuclear proteins. a, Functional annotation of proteins containing NAM-upregulated acetylation sites in HeLa cells. Significantly enriched GO biological process (GOBP) terms associated with nuclear processes are indicated. Grey bars: all sites identified; black bars: upregulated sites b, The scatter plot shows the correlation between NAMregulated acetylation in MV4-11 and HeLa cells. Correlation was determined with Pearson correlation coefficient. c, The scatter plots show the correlation between acetylation sites quantified in biological replicate experiments for NAM treated WT MEFs, Sirt $1^{-/-}$MEFs, as well as between these two conditions. Correlation was determined with Pearson correlation coefficient. d, Functional annotation of proteins with NAM- or SIRT1-upregulated acetylation sites in MEF cells. Significantly enriched GO terms are indicated. Heatmap represents p-values for each term in comparison to whole mouse proteome. The numbers within boxes indicate hyperacetylated proteins covering the percent of proteins associated with the indicated GO terms.

\section{Figure 4 | Tubacin and bufexamac-mediated increase in protein acetylation is likely mediated}

by HDAC6. a, The scatter plot shows the correlation between acetylation sites quantified in tubacinand bufexamac-treated HeLa cells. Correlation was determined with Pearson correlation coefficient. b, Verification of Hdac6 deletion in knockout MEFs. Expression of HDAC6 and acetylation of tubulin was analyzed by immunoblotting. c, The scatter plots show the correlation between acetylation sites identified in biological replicate experiments of tubacin- or bufexamac-treated MEF cells and $\mathrm{Hdac}^{-{ }^{-}}$cells, as well as between each condition. Correlation was determined by Pearson correlation coefficient. d, Functional annotation of proteins with upregulated acetylation sites in bufexamac-, tubacin treated MEFs, or in Hdac6 ${ }^{-/}$EFs. Significantly enriched GO terms are indicated. Heatmap represents p-values for each GO term in comparison to whole mouse proteome. The numbers within boxes indicate percent of hyperacetylated proteins associated with the indicated GO terms.

\section{Figure 5 | Bufexamac inhibits KDACs at lower concentrations and causes hypoxia-like} responses at higher concentrations. a, Treatment of HeLa cells with $1 \mathrm{mM}$ bufexamac results in the induction of HIF1- $\alpha$, as determined by immunoblotting. The known hypoxia mimetics CPX and DFX were used as positive controls; vinculin served as loading control. b, Verification of bufexamac-specific upregulation of HIF1- $\alpha$. HIF1- $\alpha$ protein expression was analyzed $4 \mathrm{~h}$ after treatment of cells with the indicated compounds. c, Time-course analysis of HIF1- $\alpha$ induction by bufexamac. The induction of HIF1- $\alpha$ protein was analyzed by immunoblotting. The graph shows rapid stabilization of HIF1- $\alpha$ upon bufexamac treatment with half maximal intensity $\mathrm{I}_{1 / 2}$ of about 75 min. Error bars represent SD of three independent experiments. d, HIF1- $\alpha$ is only stabilized at higher 
concentrations of bufexamac and accumulates in the nucleus. Induction of endogenous HIF1- $\alpha$ and its nuclear accumulation was analyzed using immunofluorescence microscopy; white bar: $10 \mu \mathrm{m}$. e, Bufexamac increases transcriptional activity of HIF1- $\alpha$. Luciferase activity was measured in HeLa cells transfected with HRE-Luciferase reporter upon treatment with CPX, DFX or bufexamac. Error bars represent SD of three independent experiments. $\mathbf{f}$, Bufexamac treatment increases expression of HIF1- $\alpha$ target vascular endothelial growth factor A. Vegfa mRNA levels were assessed by real-time PCR and the data were normalized to Gapdh. Error bars represent SD of two independent experiments. g, Dose-response curves of bufexamac treated cells and the corresponding immunoblots are shown (HIF-1 $\alpha$ and acetyl-tubulin were visualized simultaneously by using a mixture of antiHIF-1 $\alpha$ and K40-acetyl-tubulin antibodies). Error-bars represent SD from 3 independent experiments. Lower panel shows immunofluorescence images of HeLa cells treated with $50 \mu \mathrm{M}$ and $250 \mu \mathrm{M}$ bufexamac and immunostained for tubulin acetylation. white bar: $10 \mu \mathrm{m}$. h, Dose-response plot showing HIF1- $\alpha$ protein levels and a representative corresponding immunoblot. Cells were treated with $250 \mu \mathrm{M}$ bufexamac and with the indicated concentrations of $\mathrm{FeCl}_{3}$. Data were normalized to the maximal HIF1- $\alpha$ expression; error bars represent SD from three independent experiments. i, Iron supplementation dose-dependently inhibits bufexamac-induced HIF1- $\alpha$ transcriptional activity. Cells were transfected with a HRE-Luciferase reporter plasmid and luciferase activity was measured after treatment with $250 \mu \mathrm{M}$ bufexamac and different concentrations of $\mathrm{FeCl}_{3}$ as indicated. Error bars represent SD of three independent experiments. CPX, ciclopirox olamine; DFX, deferoxamine; HRE, hypoxia response element; Gapdh, glycerol aldehyde 3 phosphate dehydrogenase 